\title{
MIXING CONSTRUCTIONS WITH INFINITE INVARIANT MEASURE AND SPECTRAL MULTIPLICITIES
}

\author{
Alexandre I. Danilenko and Valery V. Ryzhikov
}

\begin{abstract}
We introduce high staircase infinite measure preserving transformations and prove that they are mixing under a restricted growth condition. This is used to (i) realize each subset $M \subset \mathbb{N} \cup\{\infty\}$ as the set of essential values of the multiplicity function for the Koopman operator of a mixing ergodic infinite measure preserving transformation, (ii) construct mixing power weakly mixing infinite measure preserving transformations, (iii) construct mixing Poissonian automorphisms with a simple spectrum, etc.
\end{abstract}

\section{INTRODUCTION}

Let $T$ be an invertible measure preserving transformation of a $\sigma$-finite measure space $(X, \mathfrak{B}, \mu)$ and $\mu(X)=\infty$. By $U_{T}$ we denote the Koopman unitary operator associated with $T$ :

$$
U_{T} f=f \circ T, \quad f \in L^{2}(X, \mu) .
$$

The set of essential values of the spectral multiplicity function of $U_{T}$ will be denoted by $\mathcal{M}(T)$. We note that $\mathcal{M}(T)$ is a non-empty subset of $\mathbb{N} \cup\{\infty\}$. In our previous paper $[\mathrm{DaR}]$ we showed that for each subset $M \subset \mathbb{N}$, there exists an ergodic conservative $T$ such that $\mathcal{M}(T)=M$. The main purpose of the present paper is to sharpen (and generalize) this result. We will show that $T$ can be chosen mixing or, equivalently, of zero type (see [Aa], [DaS2]). This means that $U_{T}^{n} \rightarrow 0$ weakly as $n \rightarrow \infty$.

Theorem 0.1. Given any subset $M \subset \mathbb{N} \cup\{\infty\}$, there exists a mixing ergodic conservative infinite measure preserving transformation $T$ such that $\mathcal{M}(T)=M$.

We first consider the case where $M$ does not contain $\{\infty\}$. The idea of the proof is to consider the Cartesian product $S \times T$, where $S$ is a rigid transformation with $\mathcal{M}(S)=M$ (constructed in $[\mathrm{DaR}]$ ) and $T$ is a mixing transformation such that $\mathcal{M}(S \times T)=\mathcal{M}(S)$ and $S \times T$ is ergodic. We note that the Cartesian product of a mixing system with any other (even non-ergodic) system is mixing. We construct $T$ as a certain limit of a sequence of transformations $\left(T_{n}\right)_{n>0}$ satisfying $U_{T_{n}}^{H_{k}^{(n)}} \rightarrow$ $\delta_{n} U_{T_{n}}$ weakly as $k \rightarrow \infty$ along a subsequence $H_{k}^{(n)}$ of rigidity for $S$, where $\delta_{n}$ is a sequence of positive reals tending to 0 . The transformations $T_{n}$ are not mixing of course. However they have a mixing part which occupies more and more space as $n \rightarrow \infty$. In the limit it fills the entire space. We thus consider this construction as some forcing of mixing. It remains to find a model for the mixing parts. In the case of probability preserving systems, staircase transformations [Ad] played this role (see [Ry1], [Ry2], [Ag], [Da4]). Therefore it seems natural to use "infinite" 
staircase systems for our purposes. Hence our first step is to show that the infinite staircases are mixing. Unfortunately, the restricted growth condition on the sequence of cuts essentially used by Adams in [Ad] is incompatible with the infiniteness of the invariant measure. That is why instead of pure staircases we introduce socalled high staircases. Geometrically this means that on each step of the inductive cut-and-stack construction we insert a layer of spacers between the tower and the staircase roof. If the layers are sufficiently thick, the corresponding high staircase transformation preserves an infinite measure. At the same time an analogue of the restricted growth condition can hold for high staircases (see Definition 2.3 below). Modifying Adams' argument from [Ad] we show the following theorem, which is of independent interest.

Theorem 0.2. Under the restricted growth condition each infinite measure preserving high staircase transformation is mixing.

We then utilize the transformations from Theorem 0.2 to prove Theorem 0.1 in the aforementioned way. The case where $M \ni \infty$ comes to the above by considering a product $T \times B$, where $B$ is a Bernoulli shift and $T$ is a transformation from the claim of Theorem 1 with $\mathcal{M}(T)=M \backslash\{\infty\}$. This idea 'works' if the maximal spectral type of $T$ is singular. We show that such $T$ exists.

As a byproduct we can solve an open problem related to weak mixing for infinite measure preserving systems. A transformation $T$ is called power weakly mixing if for each finite integer sequence $\left(n_{1}, \ldots, n_{k}\right), n_{i} \neq 0$ for all $i=1, \ldots, k$, the product $T^{n_{1}} \times \cdots \times T^{n_{k}}$ is ergodic. A number of rank-one power weakly mixing transformations with exotic properties are known so far [AFS], [Da1], [Da2], [DaS1] (see also surveys [Da3], [DaS2] and reference therein). However all of them are either rigid or partially rigid. Hence a problem arises:

is there a mixing power weakly mixing rank-one infinite measure preserving map? We answer affirmatively by showing the following theorem.

Theorem 0.3. There is a power weakly mixing high staircase transformation satisfying the restricted growth condition.

We conjecture that every high staircase transformation is power weakly mixing.

The method of forcing mixing originated in [Ry1] was used in [Ry2] and [Ag] to construct a mixing rank-one finite measure preserving transformations $T$ such that the unitary

$$
\exp \left(\widehat{U}_{T}\right):=\bigoplus_{n=0}^{\infty} \widehat{U}_{T}^{\odot n}
$$

has a simple spectrum. Here $\widehat{U}_{T}$ denotes the restriction of $U_{T}$ onto the orthocomplement to the constant functions. We establish an infinite version of this result.

Theorem 0.4. There is a mixing rank-one conservative infinite measure preserving transformation $T$ such that the unitary operator $\exp \left(U_{T}\right)$ has a simple spectrum.

This theorem has some applications to the theory of Poissonian automorphisms (see [CFS], [Ne], [Ro1], [Ro2]).

Corollary 0.5. There is a mixing (of all orders) Poisson suspension with a simple spectrum. 
If a Poissonian automorphism does not have a simple spectrum then the set of its spectral multiplicities is infinite. We construct examples of mixing Poisson automorphisms $S$ such that $\mathcal{M}(S)=\left\{p, p^{2}, p^{3}, \ldots\right\}$ (for an arbitrary $p>1$ ), and also $\mathcal{M}(S)=\{1,3,3 \cdot 5,3 \cdot 5 \cdot 7, \ldots\}$ (for other $S$, of course).

\section{Cut-And-Stack And $(C, F)$-COnstructions}

To prove Main Theorem we will use the $(C, F)$-construction (see [dJ], [Da1], [Da3]). We now briefly outline its formalism. Let two sequences $\left(C_{n}\right)_{n>0}$ and $\left(F_{n}\right)_{n \geq 0}$ of finite subsets in $\mathbb{Z}$ are given such that:

- $F_{n}=\left\{0,1, \ldots, h_{n}-1\right\}, \# C_{n}>1,0 \in C_{n}$,

$-F_{n}+C_{n+1} \subset F_{n+1}$,

$-\left(F_{n}+c\right) \cap\left(F_{n}+c^{\prime}\right)=\emptyset$ if $c \neq c^{\prime}, c, c^{\prime} \in C_{n+1}$,

$-\lim _{n \rightarrow \infty} \frac{h_{n}}{\# C_{1} \cdots \# C_{n}}=\infty$.

Let $X_{n}:=F_{n} \times C_{n+1} \times C_{n+2} \times \cdots$. Endow this set with the (compact Polish) product topology. The following map

$$
\left(f_{n}, c_{n+1}, c_{n+2}, \ldots\right) \mapsto\left(f_{n}+c_{n+1}, c_{n+2}, \ldots\right)
$$

is a topological embedding of $X_{n}$ into $X_{n+1}$. We now set $X:=\bigcup_{n \geq 0} X_{n}$ and endow it with the (locally compact Polish) inductive limit topology. Given $A \subset F_{n}$, we denote by $[A]_{n}$ the following cylinder: $\left\{x=\left(f, c_{n+1}, \ldots,\right) \in X_{n} \mid f \in A\right\}$. Then $\left\{[A]_{n} \mid A \subset F_{n}, n>0\right\}$ is the family of all compact open subsets in $X$. It forms a base of the topology on $X$.

Let $\mathcal{R}$ stand for the tail equivalence relation on $X$ : two points $x, x^{\prime} \in X$ are $\mathcal{R}$ equivalent if there is $n>0$ such that $x=\left(f_{n}, c_{n+1}, \ldots\right), x^{\prime}=\left(f_{n}^{\prime}, c_{n+1}^{\prime}, \ldots\right) \in X_{n}$ and $c_{m}=c_{m}^{\prime}$ for all $m>n$. There is a non-atomic Borel infinite $\sigma$-finite measure $\mu$ on $X$ which is invariant (and ergodic) under $\mathcal{R}$ and such that $\mu\left(X_{0}\right)=h_{0}$. It is unique up to scaling.

Now we define a transformation $T$ of $(X, \mu)$ by setting

$$
T\left(f_{n}, c_{n+1}, \ldots\right):=\left(1+f_{n}, c_{n+1}, \ldots\right) \text { whenever } f_{n}<h_{n}-1, n>0
$$

This formula defines $T$ partly on $X_{n}$. When $n \rightarrow \infty, T$ extends to the entire $X$ (minus countably many points) as a $\mu$-preserving invertible transformation. Moreover, the $T$-orbit equivalence relation coincides with $\mathcal{R}$ (on the subset where $T$ is defined). We call $T$ the $(C, F)$-transformation associated with $\left(C_{n+1}, F_{n}\right)_{n \geq 0}$. Below we will often use the following simple formulae

$$
\begin{gathered}
{[A]_{n} \cap[B]_{n}=[A \cap B]_{n}, \quad[A]_{n} \cup[B]_{n}=[A \cup B]_{n}} \\
{[A]_{n}=\bigsqcup_{c \in C_{n+1}}[A+c]_{n+1}, \quad \mu\left([A+c]_{n+1}\right)=\frac{\mu\left([A]_{n}\right)}{\# C_{n+1}}} \\
T^{r}[A]_{n}=[r+A]_{n}
\end{gathered}
$$

for all subsets $A, B \subset F_{n}$ and $r \in \mathbb{Z}$ such that $r+A \subset F_{n}$.

We note that in a similar way we can define $(C, F)$-actions of an arbitrary countable discrete amenable group. In that case $\left(F_{n}\right)_{n \geq 0}$ must be a $\mathrm{F} \varnothing$ lner sequence in the group. The formulae (1-1) are all satisfied for arbitrary $(C, F)$-actions. In this 
paper we are mainly interested in $\mathbb{Z}$-actions. While proving Theorem 0.3 we will also need $(C, F)$-actions of $\mathbb{Z}^{d}$ which are Cartesian products of a fixed $\mathbb{Z}$-action. A useful observation is that if $T$ is associated with $\left(C_{n+1}, F_{n}\right)_{n \geq 0}$ then the product $\mathbb{Z}^{d}$-action $\left(T^{n_{1}} \times \cdots \times T^{n_{d}}\right)_{\left(n_{1}, \ldots, n_{d}\right) \in \mathbb{Z}^{d}}$ is associated with $\left(C_{n+1}^{d}, F_{n}^{d}\right)_{n \geq 0}$, where the upper index $d$ denotes the Cartesian power.

Another observation is that the $(C, F)$-construction is equivalent to the classical cut-and-stack construction of rank-one transformations. Indeed, $X_{n}$ can be interpreted as the $n$-th tower consisting of the levels $[f]_{n}, f \in F_{n}$. It is cut into $\# C_{n+1}$ subtowers $\left[F_{n}+c\right]_{n+1}, c \in C_{n+1}$, which are then stack (with some spacers in-between) into a new, $(n+1)$-tower. $C_{n+1}$ is the set of locations of these subtowers inside the $(n+1)$-tower. More precisely, if we order the elements of $C_{n+1}$ as follows

$$
0=c(0)<\cdots<c\left(r_{n}-1\right)
$$

then $c(i)$ is exactly the hight of the bottom level of the $(i+1)$-th subtower of $X_{n}$ inside $X_{n+1}, i=0, \ldots, r_{n}-1$. That is why in the following we will illustrate some abstract aspects of the $(C, F)$-construction with more common cut-and-stack pictures.

\section{High STAircase CONSTRUCTION}

In this section we prove Theorems 0.2 and 0.3 . We first formulate a couple of definitions.

Definition 2.1. Let $T$ be a measure preserving transformation of an infinite $\sigma$ finite measure space $(X, \mathfrak{B}, \mu)$.

(i) A sequence of positive integers $a_{n}$ is called mixing for $T$ if $\mu\left(T^{a_{n}} A \cap B\right) \rightarrow 0$ as $n \rightarrow \infty$ for all subsets $A, B \subset X$ of finite measure.

(ii) A sequence of intervals $\left[a_{n}, b_{n}\right) \subset \mathbb{N}$ is called mixing for $T$ if each sequence $d_{n}$ such that $a_{n} \leq d_{n}<b_{n}$ is mixing for $T$.

Let $\left(z_{n}\right)_{n=1}^{\infty}$ and $\left(r_{n}\right)_{n=0}^{\infty}$ be two sequences of positive integers and $r_{n} \rightarrow \infty$ as $n \rightarrow \infty$. We define $\left(C_{n}\right)_{n=1}^{\infty}$ and $\left(F_{n}\right)_{n=0}^{\infty}$ inductively by setting

$$
\begin{aligned}
& h_{0} \quad \text { is arbitrary, } \\
& h_{n+1}:=r_{n}\left(h_{n}+z_{n}\right)+r_{n}\left(r_{n}-1\right) / 2, \\
& c_{n+1}(0):=0, \quad c_{n+1}(i+1):=c_{n+1}(i)+h_{n}+z_{n}+i, \\
& C_{n+1}:=\left\{c_{n+1}(i) \mid i=0, \ldots, r_{n}-1\right\}, \\
& F_{n+1}:=\left\{0, \ldots, h_{n+1}-1\right\} .
\end{aligned}
$$

Definition 2.2. The $(C, F)$-transformation $T$ on a standard $\sigma$-finite measure space $(X, \mathfrak{B}, \mu)$ associated with $\left(C_{n+1}, F_{n}\right)_{n>0}$ will be called a high staircase (see Figure 2.1). If $z_{n}=0$ for all $n$, we call $T$ a pure staircase.

Definition 2.3. By the restricted growth condition we mean the following:

$$
\lim _{n \rightarrow \infty} \frac{r_{n}^{2}}{r_{0} r_{1} \cdots r_{n-1}}=0
$$




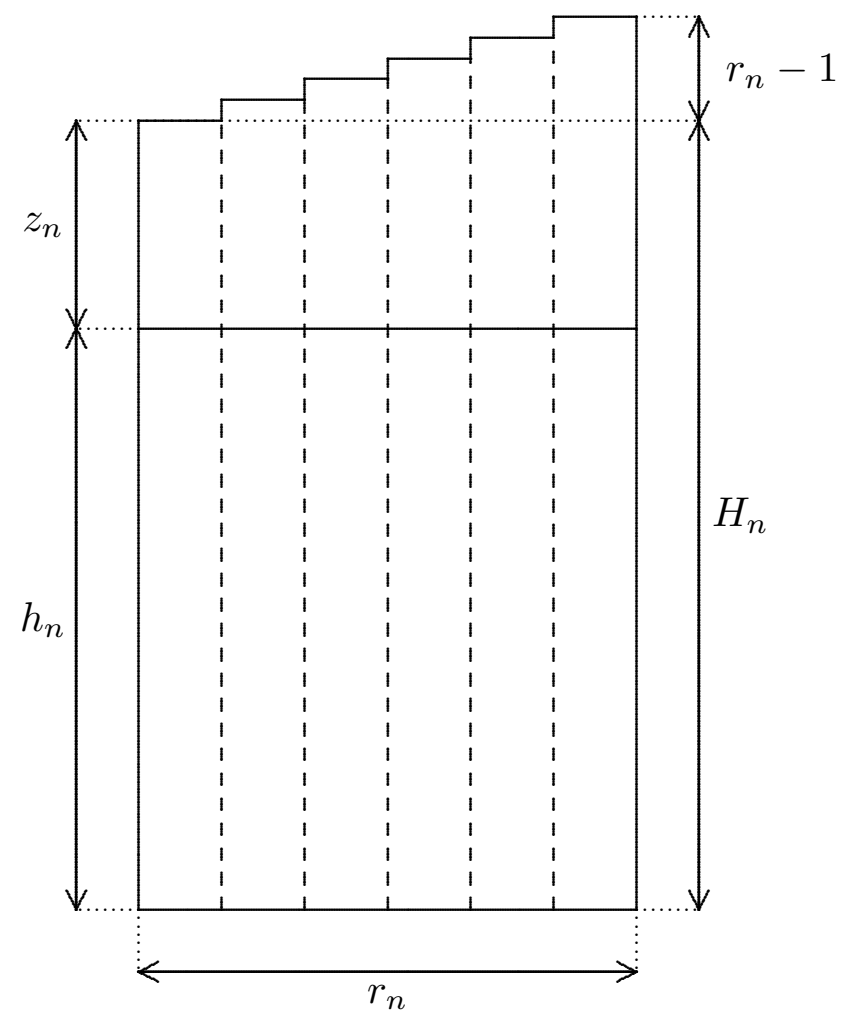

FiguRE 2.1. High staircase.

We note that this condition implies

$$
\frac{r_{n}^{2}}{h_{n}}=\frac{r_{n}^{2}}{\mu\left(X_{n}\right)} \cdot \frac{1}{r_{0} \cdots r_{n-1}} \rightarrow 0 \quad \text { as } n \rightarrow \infty \text {. }
$$

Moreover, the restricted growth condition is equivalent to (2-1) in the case when $\mu$ is finite. We remark that Adams originally introduced it for the finite measure preserving pure staircases in the form of (2-1) [Ad].

We leave to the reader the proof of the following two simple lemmata. The second one is an $L^{2}$-version of [Ad, Lemma 2.1].

Lemma 2.4. Under the restricted growth condition a high staircase is infinite measure preserving if and only if $\sum_{n=1}^{\infty} z_{n} / h_{n}=\infty$.

Lemma 2.5. Given positive integers $R, L, r$ and a measurable set $B \subset X$ of finite measure, we have

$$
\left\|\frac{1}{R} \sum_{i=0}^{R-1} U_{T}^{-i} 1_{B}\right\|_{2} \leq\left\|\frac{1}{L} \sum_{i=0}^{L-1} U_{T}^{-i r} 1_{B}\right\|_{2}+\frac{r L}{R} \sqrt{\mu(B)} .
$$

From now on we assume that $T$ is infinite measure preserving (see Lemma 2.4). The following statement is a slight modification of [Ad, Lemma 2.2].

Lemma 2.6. Let $\left[\alpha_{n}, \beta_{n}\right)$ be a sequence of intervals in $\mathbb{Z}$ which is mixing for each power of $T$ and $l_{n} \rightarrow \infty$. Take $k_{n} \in\left[\alpha_{n}, \beta_{n}\right)$. Then $\frac{1}{l_{n}} \sum_{i=0}^{l_{n}-1} U_{T}^{-k_{n} i} \rightarrow 0$ strongly as $n \rightarrow \infty$. 
Proof. Take a measurable subset $B \subset X, \mu(B)<1$. Then for each $l>0$,

$$
\left\|\frac{1}{l} \sum_{i=0}^{l-1} U_{T}^{-i k_{n}} 1_{B}\right\|_{2}^{2}=\frac{\left\|1_{B}\right\|_{2}^{2}}{l}+\frac{1}{l^{2}} \sum_{i \neq j=0}^{l-1} \mu\left(T^{(i-j) k_{n}} B \cap B\right) .
$$

Since $\mu\left(T^{p k_{n}} B \cap B\right) \rightarrow 0$ for each $p \neq 0$ by the assumption of the lemma, there is $l$ such that

$$
\left\|\frac{1}{l} \sum_{i=0}^{l-1} U_{T}^{-i k_{n}} 1_{B}\right\|_{2}^{2}<\epsilon
$$

for all sufficiently large $n$. It remains to apply Lemma 2.5.

We now let $H_{n}:=h_{n}+z_{n}$. The following lemma is crucial in the proof of Theorem 0.2 .

Lemma 2.7. If the restricted growth condition is satisfied then the sequence of intervals $\left[h_{n}, 2 H_{n}\right)$ is mixing for each non-zero power of $T$.

Proof. Fix $j>0$. We first show that the sequence $\left(H_{n}\right)_{n>0}$ is mixing for $T^{j}$. Take subsets $A, B \subset F_{n}$. Since

$$
j H_{n}+c_{n+1}(i)=c_{n+1}(i+j)-j i-\frac{j(j-1)}{2} \text { if } i+j<r_{n},
$$

we have

$$
\begin{aligned}
\mu\left(T^{j H_{n}}[A]_{n} \cap[B]_{n}\right) & =\sum_{i=0}^{r_{n}-1} \mu\left(T^{j H_{n}}\left[A+c_{n+1}(i)\right]_{n+1} \cap[B]_{n}\right) \\
& =\sum_{i=0}^{r_{n}-j-1} \mu\left(\left[-i j+A_{j}+c_{n+1}(i+j)\right]_{n+1} \cap[B]_{n}\right)+\bar{o}(1) \\
& =\frac{1}{r_{n}} \sum_{i=0}^{r_{n}-j-1} \mu\left(T^{-i j}\left[A_{j}\right]_{n} \cap[B]_{n}\right)+\bar{o}(1),
\end{aligned}
$$

where $A_{j}:=(A-j(j-1) / 2) \cap F_{n}$. We note that $\bar{o}(1)$ here and below means "uniformly small" over all $A, B \subset F_{n}$ as $n \rightarrow \infty$. Since $T$ is ergodic, $r_{n}^{-1} \sum_{i=0}^{r_{n}-1} U_{T}^{-i} \rightarrow 0$ strongly. It follows that $r_{n}{ }^{-1} \sum_{i=0}^{r_{n}-1} U_{T}^{-i j} \rightarrow 0$ strongly. Hence we are done.

Now take $a_{n} \in\left[h_{n}, 2 H_{n}\right)$. We are going to prove that $\left(a_{n}\right)_{n>0}$ is mixing for $T^{j}$. Dropping to a subsequence we may assume without loss of generality that $j a_{n}=k H_{n}+b_{n}$ for some $0 \leq b_{n}<H_{n}$ and $0 \leq k \leq 2 j$.

Suppose first that $k \neq 0$. Partition $A$ into three subsets $A_{1}, A_{2}, A_{3}$ such that $b_{n}+A_{1} \subset F_{n}, b_{n}+A_{2} \subset H_{n}+F_{n}$ and $b_{n}+A_{3} \subset\left[h_{n}, H_{n}\right)$. We verify mixing separately on each of these subsets. We note first that $T^{b_{n}}\left[A_{3}\right]_{n} \cap[B]_{n}=\emptyset$, $\mu\left([B]_{n} \triangle T^{-k H_{n}}[B]_{n}\right)=\bar{o}(1)$ and hence

$$
\mu\left(T^{j a_{n}}\left[A_{3}\right]_{n} \cap[B]_{n}\right)=\mu\left(T^{b_{n}}\left[A_{3}\right]_{n} \cap T^{-k H_{n}}[B]_{n}\right)=\bar{o}(1) .
$$

To verify mixing on on $A_{1}$ and $A_{2}$ it is enough to notice that

$$
\begin{aligned}
& j a_{n}+A_{1}=k H_{n}+\left(b_{n}+A_{1}\right) \\
& j a_{n}+A_{2}=(k+1) H_{n}+\left(b_{n}+A_{2}-H_{n}\right),
\end{aligned}
$$


$\left(b_{n}+A_{1}\right) \cup\left(b_{n}+A_{2}-H_{n}\right) \subset F_{n}$ and use the fact that the sequence $\left(H_{n}\right)_{n>0}$ is mixing for both $T^{k}$ and $T^{k+1}$.

Now consider the second case when $k=0$. Then $b_{n} \geq j h_{n}$. Hence we can partition $A$ into subsets $A_{2}$ and $A_{3}$ such that $b_{n}+A_{3} \subset\left[h_{n}, H_{n}\right)$ and $b_{n}+A_{2} \subset$ $H_{n}+F_{n}$. Therefore $T^{j a_{n}}\left[A_{3}\right]_{n} \cap[B]_{n}=\emptyset$ and

$$
\mu\left(T^{j a_{n}}\left[A_{2}\right]_{n} \cap[B]_{n}\right)=\mu\left(T^{H_{n}}\left[b_{n}-H_{n}+A_{2}\right]_{n} \cap[B]_{n}\right) \rightarrow 0
$$

because $\left(H_{n}\right)_{n=1}^{\infty}$ is mixing for $T$.

Proof of Theorem 0.2. It is enough to prove that any sequence $\left(m_{n}\right)_{n=1}^{\infty}$ such that $H_{n} \leq m_{n}<H_{n+1}$ for all $n$ is mixing (or it contains a mixing subsequence). We find integers $k_{n}$ and $t_{n}$ such that

$$
m_{n}=k_{n} H_{n}+t_{n}
$$

and $1 \leq k_{n} \leq r_{n}$ and $0 \leq t_{n}<H_{n}$. We set

$$
\begin{aligned}
& C_{n+1}^{1}:=\left\{c_{n+1}(i) \mid r_{n}-k_{n} \leq i<r_{n}\right\}, \\
& C_{n+1}^{2}:=\left\{c_{n+1}(i) \mid 0 \leq i<r_{n}-k_{n}\right\} .
\end{aligned}
$$

Mixing on $F_{n}+C_{n+1}^{1}$. This corresponds to the domain $D_{4}$ on Picture 2.2. Given $A, B \subset F_{n}$, we note that for each $0 \leq j<r_{n+1}-1$

$$
m_{n}+A+C_{n+1}^{1}+c_{n+2}(j)=D-j+c_{n+2}(j+1),
$$

where $D:=t_{n}+A+C_{n+1}^{1}-H_{n+1}$. It is important to notice that the subset $D-j$ is contained essentially in $F_{n+1}$, i.e. $\mu\left(\left[(D-j) \cap F_{n+1}\right]_{n+1}\right)=\mu\left([D]_{n+1}\right)+\bar{o}(1)$. Hence

$$
\mu\left(T^{m_{n}}\left[A+C_{n+1}^{1}\right]_{n+1} \cap[B]_{n}\right)=\frac{1}{r_{n+1}} \sum_{j=0}^{r_{n+1}-2} \mu\left(T^{-j}[D]_{n+1} \cap[B]_{n}\right)+\bar{o}(1) \rightarrow 0 .
$$

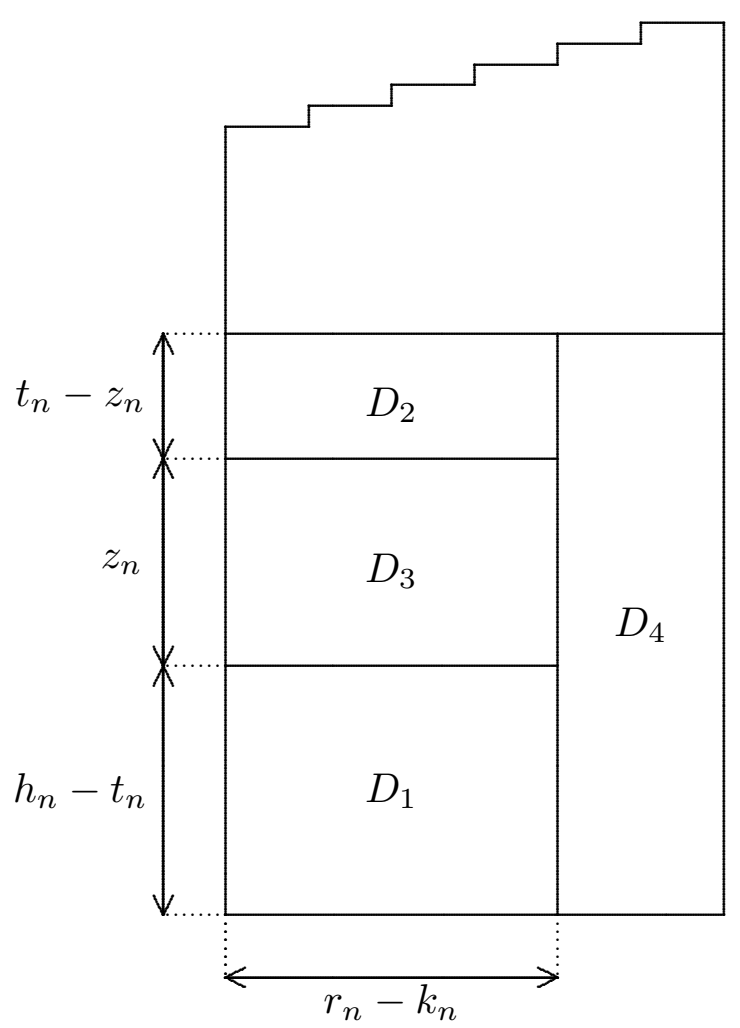

Figure 2.2. Partition of the $n$-tower into main domains. 
Mixing on $F_{n}+C_{n+1}^{2}$. Dropping to a further subsequence we can assume that $k_{n} / r_{n} \rightarrow \delta_{2}$ for some $\delta_{2}<1$. Indeed, if $k_{n} / r_{n} \rightarrow 1$ then

$$
\max _{D \subset F_{n}} \mu\left(\left[D+C_{n+1}^{2}\right]_{n+1}\right) / \mu\left([D]_{n}\right) \rightarrow 0
$$

and mixing on $F_{n}+C_{n+1}^{2}$ follows immediately. Now take $c_{n+1}(i) \in C_{n+1}^{2}$. We have

$$
m_{n}+c_{n+1}(i)=c_{n+1}\left(i+k_{n}\right)-k_{n} i-\frac{k_{n}\left(k_{n}-1\right)}{2}+t_{n} .
$$

Consider subsets $A, B \subset F_{n}$ such that $\mu\left([A \cup B]_{n}\right)<1$. Partition $A$ into three subsets $A_{1}, A_{2}, A_{3}$ such that $A_{1}+t_{n} \subset F_{n}, A_{2}+t_{n} \subset H_{n}+F_{n}$ and $A_{3}+t_{n} \subset\left[h_{n}, H_{n}\right)$. As for the graphical interpretation, $A_{i}$ is the part of $A$ that lays inside the domain $D_{i}, 1 \leq i \leq 3$, on Figure 2.2.

As in the proof of Lemma 2.7, we verify mixing separately on every of these subsets. However now $k_{n}$ may be unbounded and this fact essentially complicates the proof. The restricted growth condition yields

$$
\max _{0 \leq i<r_{n}} \max _{D \subset F_{n}}\left|\mu\left(\left[\left(D \pm k_{n} i \pm k_{n}\left(k_{n}-1\right) / 2\right) \cap D\right]_{n}\right)-\mu\left([D]_{n}\right)\right|=\bar{o}(1),
$$

i.e. we may neglect rotations by $k_{n} i+k_{n}\left(k_{n}-1\right) / 2$ inside $F_{n}$. Taking this into account we obtain mixing on $A_{3}$ in the same way as in the proof of Lemma 2.7.

Next, let us verify mixing on $A_{1}$. (Mixing on $A_{2}$ is verified in a similar way. We leave that to the reader.) Making use of (2-2) again, we obtain

$$
\mu\left(T^{m_{n}}\left[A_{1}+C_{n+1}^{2}\right]_{n+1} \cap[B]_{n}\right)=\frac{1}{r_{n}} \sum_{i=0}^{r_{n}-k_{n}-1} \mu\left(T^{-k_{n} i}\left[A_{1}^{\prime}\right]_{n} \cap[B]_{n}\right)+\bar{o}(1),
$$

where $A_{1}^{\prime}:=\left(A_{1}+t_{n}-k_{n}\left(k_{n}-1\right) / 2\right) \cap F_{n}$.

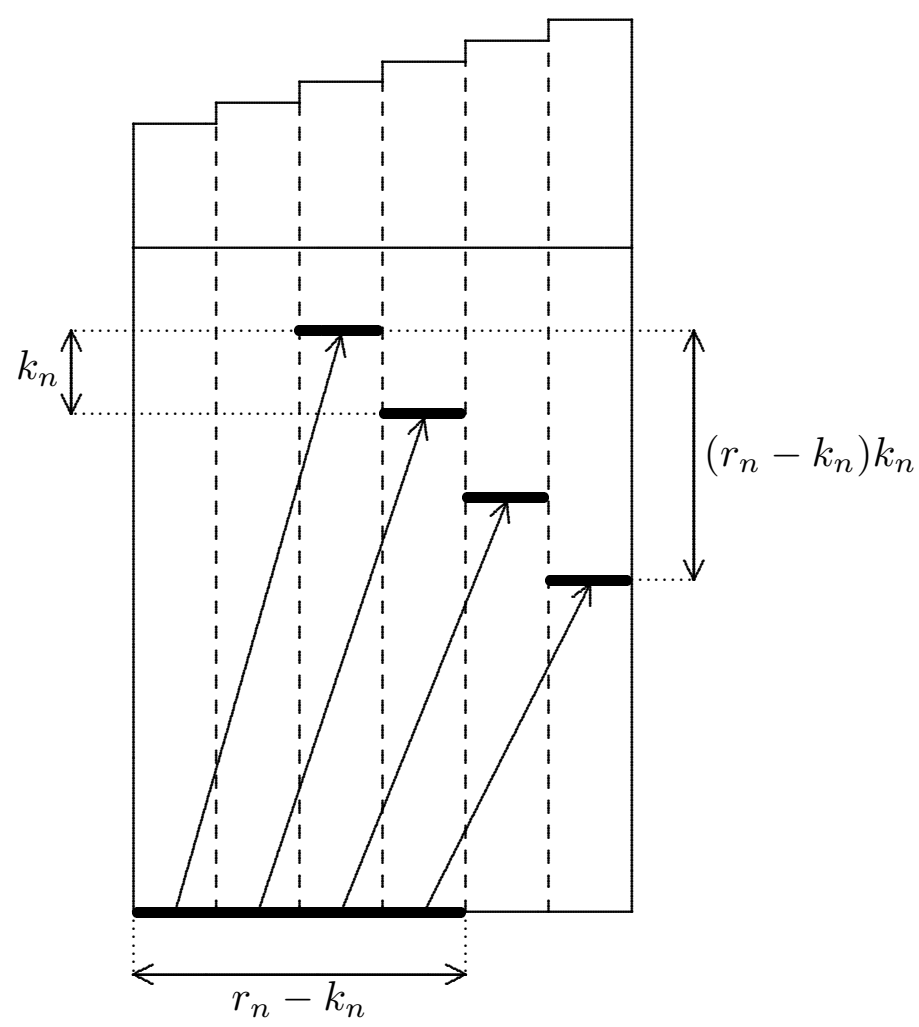

FiguRE 2.3. Image of the bottom layer of $D_{1}$ under $T^{m_{n}}$. 
There exists an integer $p_{n}$ such that

$$
h_{p_{n}} \leq\left(r_{n}-k_{n}\right) k_{n}<h_{p_{n}+1}
$$

Since $\delta_{2} \neq 1$ and $r_{n} \rightarrow \infty$, it follows that $p_{n} \rightarrow \infty$. Consider now separately three possible cases.

(A) Let $\frac{\left(r_{n}-k_{n}\right) k_{n}}{h_{p_{n}}} \rightarrow \infty$ and $k_{n} \leq h_{p_{n}}$ for all $n$. Then we can find $k_{n}^{\prime}$ such that $h_{p_{n}} \leq k_{n} k_{n}^{\prime}<2 h_{p_{n}}$. It follows that $\frac{r_{n}-k_{n}}{k_{n}^{\prime}} \rightarrow \infty$. We can choose a sequence $\left(l_{n}\right)_{n=1}^{\infty}$ so that $l_{n} \rightarrow \infty$ and $\frac{r_{n}-k_{n}}{l_{n} k_{n}^{\prime}} \rightarrow \infty$. By Lemmata 2.6 and 2.7,

$$
\frac{1}{l_{n}} \sum_{i=0}^{l_{n}-1} U_{T}^{-i k_{n} k_{n}^{\prime}} \rightarrow 0 \quad \text { strongly as } n \rightarrow \infty
$$

Now we deduce from Lemma 2.5 that

$$
\frac{1}{r_{n}} \sum_{i=0}^{r_{n}-k_{n}-1} \mu\left(T^{-k_{n} i}\left[A_{1}^{\prime}\right]_{n} \cap[B]_{n}\right) \leq\left\|\frac{1}{l_{n}} \sum_{i=0}^{l_{n}-1} U_{T}^{-k_{n} k_{n}^{\prime} i} 1_{[B]_{n}}\right\|_{2}+\frac{l_{n} k_{n}^{\prime}}{r_{n}-k_{n}} .
$$

Mixing on $A_{1}$ in this case follows from the above inequality and (2-3).

Before we pass to the remaining two cases let us show that

$$
H_{n}^{2} / h_{n+1} \rightarrow \infty
$$

Indeed,

$$
\frac{H_{n} r_{n}+r_{n}\left(r_{n}-1\right) / 2}{H_{n}^{2}} \leq \frac{r_{n}}{H_{n}}+\frac{r_{n}^{2}}{H_{n}^{2}} \leq \frac{r_{n}}{h_{n}}+\frac{r_{n}^{2}}{h_{n}^{2}} \rightarrow 0
$$

in view of $(2-1)$.

(B) Let $\frac{\left(r_{n}-k_{n}\right) k_{n}}{h_{p_{n}}} \rightarrow \infty$ and $k_{n}>h_{p_{n}}$ for all $n$. If $H_{p_{n}}<k_{n}$ for all sufficiently large $n$ then

$$
\frac{2 \delta_{2}}{1-\delta_{2}}>\frac{k_{n}^{2}}{\left(r_{n}-k_{n}\right) k_{n}}>\frac{H_{p_{n}}^{2}}{\left(r_{n}-k_{n}\right) k_{n}}>\frac{H_{p_{n}}^{2}}{h_{p_{n}+1}} \rightarrow \infty
$$

according to (2-4), a contradiction. Hence, passing to a subsequence we may assume that

$$
h_{p_{n}} \leq k_{n} \leq H_{p_{n}}
$$

for all sufficiently large $n$. Then by Lemmata 2.7 and 2.6 we deduce from (2-3) that $\mu\left(T^{m_{n}}\left[A_{1}+C_{n+1}^{2}\right]_{n+1} \cap[B]_{n}\right) \rightarrow 0$.

(C) Consider now the remaining third case where $\frac{\left(r_{n}-k_{n}\right) k_{n}}{h_{p_{n}}}$ does not go to infinity. Then dropping to a subsequence we may assume that there is $\delta_{1} \geq 1$ with

$$
\frac{\left(r_{n}-k_{n}\right) k_{n}}{h_{p_{n}}} \rightarrow \delta_{1} \quad \text { as } n \rightarrow \infty
$$

It follows that there is $\delta>0$ such that

$$
h_{p_{n}}>\delta k_{n}^{2} \quad \text { for all sufficiently large } n \text {. }
$$


We deduce from (2-4) that $H_{p_{n}-1}^{2} / h_{p_{n}} \rightarrow \infty$. This plus (2-5) imply $H_{p_{n}-1} / k_{n} \rightarrow \infty$. Hence $H_{p_{n}-1}>k_{n}>h_{p_{n}-1}$ for all sufficiently large $n$. Therefore again we can find an integer $k_{n}^{*}$ such that $H_{p_{n}-1} \leq k_{n}^{*} k_{n}<2 H_{p_{n}-1}$. The sequence of intervals $\left[H_{p_{n}}, 2 H_{p_{n}}\right)$ is mixing for all powers of $T$ by Lemma 2.7 We also have

$$
\frac{r_{n}-k_{n}}{k_{n}^{*}}=\frac{\left(r_{n}-k_{n}\right) k_{n}}{k_{n}^{*} k_{n}} \geq \frac{\left(r_{n}-k_{n}\right) k_{n}}{2 H_{p_{n}-1}} \geq \frac{\delta_{1}}{4} \cdot \frac{h_{p_{n}}}{H_{p_{n}-1}} \rightarrow \infty .
$$

Therefore arguing as in (A) we obtain mixing in the case (C).

Remark 2.8. We note that in the particular case when $z_{n} / h_{n} \rightarrow 0$ as $n \rightarrow \infty$ the proof of Theorem 0.1 is simplified significantly. It can be carried out as a slight modification of the reasoning of Adams in [Ad]. In particular, we then no longer need to consider the cases (B) and (C). Furthermore, we can "reduce" the interval $\left[h_{n}, 2 H_{n}\right)$ in the statement of Lemma 2.7 to the interval $\left[h_{n}, 2 h_{n}\right)$. Nevertheless, this particular case of Theorem 0.2 is enough to demonstrate Theorems 0.3 and 0.4. However in the proof of our main result-Theorem 0.1 - we essentially use Theorem 0.2 in the full generality. More precisely, it is utilized to satisfy (3-2) below.

Our next purpose is to construct a power weakly mixing rank-one infinite measure preserving transformation which is mixing. For that we will use high staircases and Theorem 0.2.

Proof of Theorem 0.3. We will construct these high staircases via an inductive procedure. Suppose that after $p$ steps we have already defined $F_{0}, C_{1}, F_{1}, \ldots, C_{n_{p}}, F_{n_{p}}$.

Step $p+1$. Consider an auxiliary finite measure preserving pure staircase $T_{p+1}$ associated with a sequence $\left(C_{k}^{(p+1)}, F_{k}^{(p+1)}\right)_{k \geq 0}$ such that $F_{0}^{(p+1)}:=F_{n_{p}}$. Assume that the restricted growth condition is satisfied for $T_{p+1}$. Let $\left(X^{(p+1)}, \mu_{p+1}\right)$ be the space of this action. We normalize $\mu_{p+1}$ in such a way that

$$
\mu_{p+1}\left([0]_{0}\right)=\frac{1}{\# C_{1} \cdots \# C_{n_{p}}} .
$$

Take any finite sequence $l=\left(l_{1}, \ldots, l_{p+1}\right)$ of non-negative integers such that $\|l\|:=$ $\max _{0<i \leq p+1}\left|l_{i}\right| \leq p+1$. Since $T_{p+1}$ is mixing [Ad], the transformation

$$
S_{l}:=T_{p+1}^{l_{1}} \times \cdots \times T_{p+1}^{l_{p+1}}
$$

of the product space $\left(X^{(p+1)}, \mu_{p+1}\right)^{p+1}$ is ergodic. Then there are $N_{p+1}>0$ and $M_{p+1}>0$ such that for all disjoint subsets $A, B \subset\left(F_{0}^{(p+1)}\right)^{p+1}$ of equal cardinality there exist subsets $A^{\prime} \subset[A]_{0}$ and $B^{\prime} \subset[B]_{0}$ and their partitions $A^{\prime}=\bigsqcup_{i=1}^{M_{p+1}} A_{i}$, $B^{\prime}=\bigsqcup_{i=1}^{M_{p+1}} B_{i}$ such that

$$
\begin{gathered}
\mu_{p+1}^{\times(p+1)}\left(A^{\prime}\right)>0.5 \mu_{p+1}^{\times p+1}\left([A]_{0}\right), \quad \mu_{p+1}^{\times p+1}\left(B^{\prime}\right)>0.5 \mu_{p+1}^{\times p+1}\left([B]_{0}\right), \\
A_{i}, B_{i} \text { are } N_{p+1} \text {-cylinders (some may be empty) and } \\
S_{l}^{i} A_{i}=B_{i} \quad \text { for all } i=1, \ldots, M_{p+1} .
\end{gathered}
$$

It is assumed that $N_{p+1}$ and $M_{p+1}$ are common for all $S_{l}$ with $\|l\| \leq p+1$. We now set

$$
C_{n_{p}+1}:=C_{1}^{(p+1)}, F_{n_{p}+1}:=F_{1}^{(p+1)}, \ldots, F_{n_{p}+N_{p+1}}:=F_{N_{p+1}}^{(p+1)} .
$$


Next, we add several more subsets $\left(C_{i}, F_{i}\right)_{i=n_{p}+N_{p+1}+1}^{n_{p+1}}$ in the high staircase shape and such that the corresponding parameters $z_{i}$ incorporated into $C_{i}$ are large. These subsets are needed to get infinite measure in the limit. The $(p+1)$-step is now completed.

Continuing this procedure infinitely many times, we obtain the entire sequence $\left(C_{i+1}, F_{i}\right)_{i=0}^{\infty}$. Denote by $(X, \mu, T)$ the associated $(C, F)$-dynamical system. We normalize $\mu$ in such a way that $\mu\left([0]_{0}\right)=1$. By construction, $T$ is a high staircase. Since $z_{i}$ are large for infinitely many $i$, it follows that $\mu(X)=\infty$. Of course, we may assume without loss of generality that the restricted growth condition is satisfied. Hence $T$ is mixing by Theorem 0.2 .

To verify that $T$ is power weakly mixing it is enough to notice that

$$
\begin{gathered}
F_{0}^{(p+1)}=F_{n_{p}}, F_{N_{p+1}}^{(p+1)}=F_{n_{p}+N_{p+1}} \text { and } \\
\mu_{p+1}\left([A]_{N_{p+1}}\right)=\mu\left([A]_{n_{p}+N_{p+1}}\right)
\end{gathered}
$$

for each subset $A \subset F_{N_{p+1}}^{(p+1)}, p>0$, and use (2-6). We note that (2-7) follows from the normalization conditions for $\mu_{p+1}$ and $\mu$.

\section{Spectral multiplicities of MiXing infinite MEASURE PRESERVING TRANSFORMATIONS}

In this section we use Theorem 0.2 to show Theorems 0.1 and 0.4 . We first prove an auxiliary lemma about about cyclic spaces for products of unitary operators.

Lemma 3.1. Let $U, V$ be two unitary operators in a Hilbert space $\mathcal{H}$ and let $V$ has a simple spectrum. Let $\mathcal{C}$ be a $U$-cyclic space generated by a vector $h_{U}$ and let $h_{V}$ be a cyclic vector for $V$. If there is a sequence $n_{i} \rightarrow \infty$ such that $U^{n_{i}} \rightarrow I$ and $V^{n_{i}} \rightarrow a V^{*}$ weakly for some $a>0$ then $\mathcal{C} \otimes \mathcal{H}$ is the $U \otimes V$-cyclic space generated by $h_{U} \otimes h_{V}$.

Proof. Let $h_{U}$ and $h_{V}$ be cyclic vectors for $U$ and $V$ respectively. Denote by $\mathcal{D}$ the $(U \otimes V)$-cyclic space generated by $h_{U} \otimes h_{V}$. Since $(U \otimes V)^{n_{i}} \rightarrow a I \otimes V^{*}$ and $\mathcal{D}$ is invariant under the weak limits of powers of $U \otimes V$, it follows that $h_{U} \otimes V^{*} h_{V} \in \mathcal{D}$. In a similar way, $h_{U} \otimes V^{n} h_{V} \in \mathcal{D}$ for each $n \in \mathbb{Z}$. Therefore $h_{U} \otimes \mathcal{H} \subset \mathcal{D}$ which implies, in turn, that $\mathcal{C} \otimes \mathcal{H} \subset \mathcal{D}$. The converse inclusion is obvious.

We need to consider a class of transformations which is more general than the high staircases. Let three sequences of positive integers $\left(z_{n}\right)_{n=1}^{\infty},\left(r_{n}\right)_{n=1}^{\infty}$ and $\left(d_{n}\right)_{n=1}^{\infty}$ be given such that $d_{n} \leq r_{n}$ and $\lim _{n \rightarrow \infty} d_{n} / r_{n}=\delta \geq 0$. Suppose that the following are satisfied

$$
\begin{aligned}
& C_{n+1}:=\left\{c_{n+1}(i) \mid i=0, \ldots, r_{n}-1\right\} \\
& 0=c_{n+1}(0)<c_{n+1}(1)<\cdots<c_{n+1}\left(r_{n}-1\right), \\
& c_{n+1}(i+1):=c_{n+1}(i)+h_{n}+z_{n}+i-d_{n} \text { if } i=d_{n}, \ldots, r_{n}-1,
\end{aligned}
$$

We call the transformation associated with $\left(C_{n+1}, F_{n}\right)_{n \geq 0}$ a $(1-\delta)$-partially high staircase. Geometrically this means that on the $n$-th step we cut the $n$-th tower into $r_{n}$ subtowers and arrange spacers on the tops of $d_{n}$ first subtowers in an arbitrary way. On the tops of the remaining $r_{n}-d_{n}$ subtowers the spacers are arranged in 
the high staircase way. If $\delta=0$ then we call the transformation an almost high staircase. Since the proportion of the first $d_{n}$ subtowers of such a transformation goes to 0 , it can be deduced from the proof of Theorem 0.2 that every almost high staircase satisfying the restricted growth condition is mixing.

Proof of Theorem 0.1. Suppose first that $\infty \notin M$. It was shown in $[\mathrm{DaR}]$ that there exists an ergodic conservative infinite measure preserving transformation $S$ such that $\mathcal{M}(S)=M$ and $U_{S}^{i_{m}} \rightarrow I$ weakly for a certain sequence $i_{m} \rightarrow \infty$. Let $(Y, \nu)$ denote the $\sigma$-finite space where $S$ acts.

Now we will "force mixing" of such transformations along the scheme outlined in the introduction. Fix a sequence $\delta_{n} \rightarrow 0$. We will construct a sequence of $\left(1-\delta_{n}\right)$-partially high staircases $T_{n}$ associated with $\left(C_{k}^{(n)}, F_{k-1}^{(n)}\right)_{k>0}$ such that

$$
U_{T_{n}}^{H^{(n)}} \rightarrow \delta_{n} U_{T_{n}}^{*} \quad \text { weakly as } k \rightarrow \infty,
$$

where $\left(H_{k}^{(n)}\right)_{k=1}^{\infty}$ is a subsequence of $\left(i_{m}\right)_{m=1}^{\infty}$. For that we put

$$
\begin{aligned}
& C_{k+1}^{(n)}=\left\{c_{k+1}^{(n)}(i) \mid 0 \leq i<r_{k}^{(n)}\right\}, \quad \text { where } \\
& c_{k+1}^{(n)}(i+1):= \begin{cases}0, & \text { for } i=-1 \\
c_{k+1}^{(n)}(i)+H_{k}^{(n)}+1, & \text { for } 0 \leq i<d_{k}^{(n)}-1 \\
c_{k+1}^{(n)}(i)+H_{k}^{(n)}+i-d_{k}^{(n)}+1, & \text { for } d_{k}^{(n)}-1 \leq i<r_{k}^{(n)}-1\end{cases}
\end{aligned}
$$

and $H_{k}^{(n)}:=h_{k}^{(n)}+z_{k}^{(n)}$ and $F_{k+1}^{(n)}=\left[0, h_{k+1}^{(n)}\right)$, where

$$
h_{k+1}^{(n)}:=r_{k}^{(n)} H_{k+1}^{(n)}+d_{k}^{(n)}-1+\frac{\left(r_{k}^{(n)}-d_{k}^{(n)}-1\right)\left(r_{k}^{(n)}-d_{k}^{(n)}\right)}{2} .
$$

The initial heights $h_{0}^{(n)}$ (i.e. sets $F_{0}^{(n)}$ ) are not specified yet. This will be done below. We now impose the following restrictions on the parameters $r_{k}^{(n)}, d_{k}^{(n)}, z_{k}^{(n)}$ :

$$
\begin{gathered}
r_{k}^{(n)} \rightarrow \infty, \quad 2 \delta_{n} \geq d_{k}^{(n)} / r_{k}^{(n)} \rightarrow \delta_{n} \quad \text { as } k \rightarrow \infty, \\
\sum_{k=1}^{\infty} z_{k}^{(n)} / h_{k}^{(n)}=\infty \quad \text { and } \\
\left\{H_{k}^{(n)} \mid k \in \mathbb{N}\right\} \subset\left\{i_{m} \mid m \in \mathbb{N}\right\} .
\end{gathered}
$$

Several additional restrictions will appear below. To verify (3-1) take subsets $A, B \subset\left[r_{k}^{(n)}, h_{k}^{(n)}\right)$. Then up to $\bar{o}(1)$ in measure the subset $T_{n}^{H_{k}^{(n)}}[A]_{k}$ equals

$$
\bigsqcup_{i=1}^{d_{k}^{(n)}-1}\left[A-1+c_{k+1}^{(n)}(i)\right]_{k+1} \sqcup \bigsqcup_{i=1}^{r_{k}^{(n)}-d_{k}^{(n)}-1}\left[A-i+c_{k+1}^{(n)}\left(i+d_{k}^{(n)}\right)\right]_{k+1} .
$$

Hence

$$
\left\langle U_{T_{n}}^{H_{k}^{(n)}} 1_{[A]_{k}}, 1_{[B]_{k}}\right\rangle=\frac{d_{k}^{(n)}}{r_{k}^{(n)}}\left\langle U_{T_{n}}^{*} 1_{[A]_{k}}, 1_{[B]_{k}}\right\rangle+\frac{1}{r_{k}^{(n)}} \sum_{i=1}^{r_{k}^{(n)}-d_{k}^{(n)}-1}\left\langle U_{T_{n}}^{-i} 1_{[A]_{k}}, 1_{[B]_{k}}\right\rangle+\bar{o}(1)
$$


and (3-1) follows because $T_{n}$ is ergodic.

The sought-for transformation $T$ will appear as a $(C, F)$-transformation associated with a concatenated sequence

$$
F_{0}^{(1)}, C_{1}^{(1)}, \ldots, F_{k_{1}}^{(1)}, C_{1}^{(2)}, F_{1}^{(2)}, C_{2}^{(2)}, \ldots, F_{k_{2}}^{(2)}, \ldots
$$

Of course, to make this concatenation well defined we need to satisfy a compatibility condition $F_{k_{n}}^{(n)}=F_{0}^{(n+1)}$. By this condition we determine all the initial heights $h_{0}^{(n)}$ except for $h_{0}^{(1)}$ which is chosen in an arbitrary way. It remains to specify the sequence of stopping times $\left(k_{n}\right)_{n=1}^{\infty}$. This will be done inductively.

Fix a vector $v$ in $L^{2}(Y, \nu)$ and denote by $\mathcal{C}$ the $U_{S}$-cyclic subspace generated by $v$. Fix a dense countable subset $\left(v_{i}\right)_{i=1}^{\infty}$ in $\mathcal{C}$. Suppose that we have already determined $k_{1}, \ldots, k_{n-1}$. Then $F_{0}^{(n)}$ is defined by the compatibility condition. The other sets $C_{1}^{(n)}, F_{1}^{(n)}, C_{2}^{(n)}, \ldots$ are defined by the above recurrent formulae. Let $T_{n}$ be the associated $(C, F)$-transformation acting on a space $\left(X^{(n)}, \mu^{(n)}\right)$. As in the proof of Theorem 0.3 we normalize $\mu^{(n)}$ in such a way that

$$
\mu^{(n)}\left([0]_{0}\right)=\left(\prod_{i=1}^{n-1} \# C_{1}^{(i)} \cdots \# C_{k_{i}}^{(i)}\right)^{-1} .
$$

Since $T_{n}$ has a simple spectrum, we can choose a cyclic vector $w_{n}$ for $U_{T_{n}}$. Since (3-1) and (3-2) are satisfied, $v \otimes w_{n}$ is a cyclic vector for $\left(U_{S} \uparrow \mathcal{C}\right) \otimes U_{T_{n}}$ by Lemma 3.1. Hence we can select a large $k_{n}$ in such a way that there are a subset $A \subset F_{k_{n}}^{(n)}$, a function $w_{n}^{\prime}$ in the linear span of the indicators $1_{[f]_{k_{n}}}, f \in A$, and $M>0$ such that $M+A \subset F_{k_{n}}^{(n)}$ and

$$
\max _{f^{\prime} \in F_{0}^{(n)}}\left\|v_{i} \otimes 1_{\left[f^{\prime}\right]_{0}}-\sum_{j=-M}^{M} \alpha_{i, j}\left(U_{S} \otimes U_{T_{n}}\right)^{j} v \otimes w_{n}^{\prime}\right\|_{2}<\epsilon_{n}
$$

for all $i=1, \ldots, n$, where $\alpha_{i, j}$ are some real numbers and $\epsilon_{n} \rightarrow 0$ very fast. This completes the $n$-th step of the inductive procedure. On this step we defined a fragment $C_{1}^{(n)}, \ldots, F_{k_{n}}^{(n)}$ of (3-3). Continuing this infinitely many times we construct the entire sequence (3-3). Rename it as $F_{0}, C_{1}, F_{1}, \ldots$ Let $T$ be the associated $(C, F)$-transformation and let $(X, \mu)$ be the space of this transformation. The measure $\mu$ is normalized so that $\mu\left([0]_{0}\right)=1$. By the construction, $T$ is an almost high staircase and $\mu(X)=\infty$. By choosing the parameters $r_{k}^{(n)}$ in a right way we may assume without loss of generality that the restricted growth condition is satisfied for $T$. Hence $T$ is mixing.

Now we are going to show that $\mathcal{M}(S \times T)=\mathcal{M}(S)$. Let $\mathcal{H}^{(n)}$ denote the linear span of the indicators $1_{[f]_{0}}, f \in F_{0}^{(n)}$ in $L^{2}\left(X^{(n)}, \mu^{(n)}\right)$ and let $\mathcal{H}_{k}$ denote the linear span of the indicators $1_{[f]_{k}}, f \in F_{k}$, in $L^{2}(X, \mu)$. We note that $F_{k_{1}+\cdots+k_{n}}=F_{0}^{(n)}$ for all $n>0$. Moreover, it is easy to see that the natural identification

$$
1_{[f]_{k_{1}+\cdots+k_{n}}} \leftrightarrow 1_{[f]_{0}}, \quad f \in F_{k_{1}+\cdots+k_{n}}
$$

extends to a linear isomorphism of $\mathcal{H}_{k_{1}+\cdots+k_{n}}$ onto $\mathcal{H}^{(n)}$. Moreover, it is an isometry because of the normalizations imposed on $\mu$ and $\mu^{(n)}$. Hence $(3-4)$ yields the 
existence of $w_{n}^{\prime} \in \mathcal{H}_{k_{1}+\cdots+k_{n+1}}$ such that

$$
\max _{f^{\prime} \in F_{k_{1}+\cdots+k_{n}}}\left\|v_{i} \otimes 1_{\left[f^{\prime}\right]_{k_{1}+\cdots+k_{n}}}-\sum_{j=-M}^{M} \alpha_{i, j}\left(U_{S} \otimes U_{T}\right)^{j} v \otimes w_{n}^{\prime}\right\|_{2}<\epsilon_{n}
$$

for all $i=1, \ldots, n, n \in \mathbb{N}$. It follows that the subspace $\mathcal{C} \otimes L^{2}(X, \mu)$ is cyclic for $U_{S} \otimes U_{T}$.

Consider now a decomposition of $L^{2}(Y, \nu)$ into $U_{S}$-cyclic spaces $\mathcal{C}_{i, j}$ :

$$
L^{2}(Y, \nu)=\bigoplus_{i \in \mathcal{M}(S)} \bigoplus_{j=1}^{i} \mathcal{C}_{i, j}
$$

such that $\sigma_{i, j} \sim \sigma_{i, j^{\prime}}$ for all $i, j, j^{\prime}$ and $\sigma_{i, j} \perp \sigma_{i^{\prime}, j^{\prime}}$ if $i \neq i^{\prime}$, where $\sigma_{i, j}$ is a measure of maximal spectral type of $U_{S} \uparrow \mathcal{C}_{i, j}$. It follows that a subspace $C_{i, j} \oplus C_{i^{\prime}, j^{\prime}}$ is $U_{S}$-cyclic if $i \neq i^{\prime}$. Given a $U_{S}$-cyclic space $\mathcal{C}$, we showed above how to construct a mixing infinite measure preserving almost high staircase dynamical system $(X, \mu, T)$ such that $\mathcal{C} \otimes L^{2}(X, \mu)$ is $U_{S} \otimes U_{T}$-cyclic. It is clear that the construction can be obviously modified so to obtain the same for an arbitrary countable family of $U_{S}$-cyclic subspaces. In particular, we can construct a mixing infinite measure preserving almost high staircase system $(X, \mu, T)$ such that the subspaces

$$
\left(\mathcal{C}_{i, j} \oplus \mathcal{C}_{i^{\prime}, j^{\prime}}\right) \otimes L^{2}(X, \mu) \quad \text { are } U_{S} \otimes U_{T^{-c y c l i c}}
$$

for all $i \neq i^{\prime} \in \mathcal{M}(S), 1 \leq j \leq i$ and $1 \leq j \leq i^{\prime}$. Denote by $\widehat{\sigma}_{i, j}$ a measure of maximal spectral type for $\left(U_{S}\left\lceil\mathcal{C}_{i, j}\right) \otimes U_{T}\right.$. It follows from (3-5) that $\widehat{\sigma}_{i, j} \perp \widehat{\sigma}_{i^{\prime}, j^{\prime}}$ if $i \neq i^{\prime}$. Of course, $\sigma_{i, j} \sim \sigma_{i, j^{\prime}}$ for all $i, j, j^{\prime}$. Since

$$
L^{2}(Y, \nu) \otimes L^{2}(X, \mu)=\bigoplus_{i \in \mathcal{M}(S)} \bigoplus_{j=1}^{i} \mathcal{C}_{i, j} \otimes L^{2}(X, \mu)
$$

and $\mathcal{C}_{i, j} \otimes L^{2}(X, \mu)$ is $U_{S} \otimes U_{T}$-cyclic for each pair $i, j$, it follows that $\mathcal{M}(S \times T)=$ $\mathcal{M}(S)$.

Thus the theorem is "almost" proved. It only remains unclear whether $T \times S$ is ergodic or not. Therefore to obtain the desired ergodicity we will modify the construction of $T$ : we will force ergodicity for $T \times S$ simultaneously with forcing mixing for $T$. For that we alternate the above argument with the argument from the proof of Theorem 0.3. On the odd steps we construct fragments of (3-3) exactly as above to retain the desired spectral properties and mixing. It remains to explain the construction on the even steps.

Suppose we have already constructed

$$
F_{0}^{(1)}, C_{1}^{(1)}, \ldots, C_{k_{n-1}}^{(n-1)}, F_{k_{n-1}}^{(n-1)}
$$

with $n-1$ odd. Now we consider a pure staircase infinite $(C, F)$-sequence

$$
F_{0}^{(n)}, C_{1}^{(n)}, F_{1}^{(n)}, C_{2}^{(n)}, \ldots
$$


such that $F_{0}^{(n)}=F_{k_{n}-1}^{n-1}$. Recall that this means that

$$
C_{k}^{(n)}=\left\{c_{k}(i) \mid i=0, \ldots, r_{k}-1\right\}
$$

$c_{k}(0)=0, c_{k}(i+1)=c_{k}(i)+h_{k-1}+i$, where $h_{k-1}$ is the hight of $F_{k-1}^{(n)}$ and $h_{k}=r_{k} h_{k-1}+r_{k}\left(r_{k}-1\right) / 2$. We also assume that the restricted growth condition $r_{k}^{2} / h_{k} \rightarrow 0$ as $k \rightarrow \infty$ is satisfied. Let $T_{n}$ be the $(C, F)$-transformation acting on a measured space $\left(X^{(n)}, \mu^{(n)}\right)$. Notice that $\mu^{(n)}\left(X^{(n)}\right)<\infty$. As above, we normalize $\mu_{n}$ in such a way that

$$
\mu^{(n)}\left([0]_{0}\right)=\left(\prod_{i=1}^{n-1} \# C_{1}^{(i)} \cdots \# C_{k_{i}}^{(i)}\right)^{-1}
$$

By $[\mathrm{Ad}], T_{n}$ is mixing. Hence the Cartesian product $S \times T_{n}$ is ergodic [FW]. Recall that $S$ is a $(C, F)$-map $[\mathrm{DaR}]$. Let $\left(C_{m+1}^{S}, F_{m}^{S}\right)_{m=0}^{\infty}$ denote the corresponding sequence. Since $S \times T_{n}$ is ergodic, there are integers $k_{n}>0$ and $M_{n}>0$ such that for all disjoint subsets $A, B \subset F_{0}^{S} \times F_{0}^{(n)}$ of equal cardinality there exist subsets $A^{\prime} \subset[A]_{0}$ and $B^{\prime} \subset[B]_{0}$ and their partitions $A^{\prime}=\bigsqcup_{i=1}^{M_{n}} A_{i}$ and $B^{\prime}=\bigsqcup_{i=1}^{M_{n}} B_{i}$ such that

$$
\begin{gathered}
\nu \times \mu^{(n)}\left(A^{\prime}\right)>0.5\left(\nu \times \mu^{(n)}\right)\left([A]_{0}\right), \\
\nu \times \mu^{(n)}\left(B^{\prime}\right)>0.5\left(\nu \times \mu^{(n)}\right)\left([B]_{0}\right), \\
A_{i}, B_{i} \text { are } k_{n} \text {-cylinders (some may be empty) and }
\end{gathered}
$$$$
\left(S \times T_{n}\right)^{i} A_{i}=B_{i} \text { for all } i=1, \ldots, M_{n} .
$$

Then we "continue" (3-6) with the following fragment: $C_{1}^{(n)}, F_{1}^{(n)}, \ldots, C_{k_{n}}^{(n)}, F_{k_{n}}^{(n)}$.

Thus we explained the construction procedure entirely. The corresponding $(C, F)$ transformation $T$ is as desired. Indeed, we have $\mathcal{M}(S \times T)=\mathcal{M}(S)$ and $S \times T$ is mixing due to the odd steps of our construction process. Furthermore, (3-7) implies that $S \times T$ is ergodic.

Now let $\infty \in M$. Assume first that the set $M^{\prime}:=M \backslash\{\infty\}$ is nonempty. This case would immediately come to the previous one whenever we know that the maximal spectral type of $S \times T$ is singular. Unfortunately, we do not see easy ways to show this property for every $S$. However, it holds for certain $S$ that 'splits into product' of two other transformations. Thus our goal is to show that $S$ can always be chosen in this special way. For that we proceed in several steps.

Fix an ergodic conservative rigid transformation $S$ with $\mathcal{M}(S)=M^{\prime}[\mathrm{DaR}]$. We also fix a family of $S$-cyclic spaces $\mathcal{C}_{i, j}, i \in M^{\prime}, 1 \leq j \leq i$ as above.

Claim $A$. There exists an infinite measure preserving transformation $R$ of a $\sigma$ finite standard measure space $(Z, \mathfrak{F}, \kappa)$ such that

(A1) $R$ is rigid along a subsequence of $\left(i_{m}\right)_{m>0}$ (the latter is a rigidity sequence for $S$ ),

(A2) $R \times S$ is conservative and ergodic,

(A3) the subspaces $L^{2}(Z, \kappa) \otimes\left(\mathcal{C}_{i, j} \oplus \mathcal{C}_{i^{\prime}, j^{\prime}}\right)$ are $U_{R} \otimes U_{S^{-c y c l i c}}$ for all $i \neq i^{\prime} \in$ $\mathcal{M}(S), 1 \leq j \leq i$ and $1 \leq j \leq i^{\prime}$. 
Recall that the group $\operatorname{Aut}(Z, \kappa)$ of all $\mu$-preserving invertible transformations of $(Z, \kappa)$ is Polish in the weak topology [CK]. It is well known (and easy to verify) that the set $\mathcal{T}_{1}$ of all transformations $R$ satisfying $(A 1)$ is a dense $G_{\delta}$ in $\operatorname{Aut}(Z, \kappa)$. The map

$$
f_{i, i^{\prime}, j, j^{\prime}}: R \mapsto U_{R} \otimes\left(U_{S} \uparrow\left(\mathcal{C}_{i, j} \oplus \mathcal{C}_{i^{\prime}, j^{\prime}}\right)\right)
$$

from $\operatorname{Aut}(Z, \kappa)$ to the group of unitary operators (of a corresponding Hilbert space) equipped with the weak operator topology is continuous for each quadruple of indices $i, i^{\prime}, j, j^{\prime}$. Recall that the set of unitary operators with a simple continuous spectrum is a $G_{\delta}$ in the the group of all unitary operators [Na]. Hence the set $\mathcal{T}_{3}$ of all transformations $R$ satisfying $(A 3)$ is a $G_{\delta}$ in $\operatorname{Aut}(Z, \kappa)$. Since the set of ergodic conservative transformations is a $G_{\delta}$ in $\operatorname{Aut}(Z \times Y, \kappa \times \nu)[\mathrm{CK}]$ and the map $R \mapsto R \times S$ is continuous, it follows that the set $\mathcal{T}_{2}$ of all transformations $R$ satisfying $(A 2)$ is also a $G_{\delta}$ in $\operatorname{Aut}(Z, \kappa)$. As follows from the first part of the proof of Theorem 0.1 , the intersection $\mathcal{T}_{2} \cap \mathcal{T}_{3}$ is nonempty (the transformation $T$ constructed there belongs to it). Since the intersection is invariant under the conjugacy and the conjugacy class of each conservative ergodic transformation is dense in $\operatorname{Aut}(Z, \kappa)[\mathrm{CK}]$, we deduce that $\mathcal{T}_{2} \cap \mathcal{T}_{3}$ is a dense $G_{\delta}$ in $\operatorname{Aut}(Z, \kappa)$. Hence so is $\mathcal{T}_{1} \cap \mathcal{T}_{2} \cap \mathcal{T}_{3}$. The claim follows.

We deduce from Claim A that the product transformation $R \times S$ is ergodic, conservative and rigid along a subsequence of $\left(i_{m}\right)_{m>0}$. Moreover,

$$
L^{2}(Z \times Y, \kappa \times \nu)=\bigoplus_{i \in M^{\prime}} \bigoplus_{1 \leq j \leq i} L^{2}(Z, \kappa) \otimes \mathcal{C}_{i, j}
$$

and the restrictions of $U_{R \times S}$ to cyclic subspaces $L^{2}(Z, \kappa) \otimes \mathcal{C}_{i, j}$ and $L^{2}(Z, \kappa) \otimes \mathcal{C}_{i^{\prime}, j^{\prime}}$ are either unitarily equivalent if $i=i^{\prime}$ or spectrally disjoint if $i \neq i^{\prime}$. In particular, $\mathcal{M}(R \times S)=\mathcal{M}(S)=M^{\prime}$.

Claim B. There is a mixing transformation $T$ such that

(B1) $L^{2}(Z \times Y \times X, \kappa \times \nu \times \mu)=\bigoplus_{i \in M^{\prime}} \bigoplus_{1 \leq j \leq i} L^{2}(Z, \kappa) \otimes \mathcal{C}_{i, j} \otimes L^{2}(X, \mu)$,

$(B 2)$ every subspace $\mathcal{C}_{i, j}^{\prime}:=L^{2}(Z, \kappa) \otimes \mathcal{C}_{i, j} \otimes L^{2}(X, \mu)$ is $U_{R \times S \times T}$-cyclic,

(B3) the unitary operators $U_{R \times S \times T} \uparrow \mathcal{C}_{i, j}^{\prime}$ and $U_{R \times S \times T} \uparrow \mathcal{C}_{i^{\prime}, j^{\prime}}^{\prime}$ are either unitarily equivalent if $i=i^{\prime}$ or spectrally disjoint if $i \neq i^{\prime}$.

(B4) $R \times S \times T$ is ergodic and conservative.

This claim can be shown by repeating almost verbally the argument from the first part of the proof of Theorem 0.1 (for $M$ without $\infty$ ). One should just replace $S$ with $R \times S$.

The following assertion is well known. We state it without proof.

Claim $C$. If $V$ and $W$ are unitary operators acting in infinite dimensional Hilbert spaces $\mathcal{H}_{V}$ and $\mathcal{H}_{W}$ such that $\infty \notin \mathcal{M}(V \otimes W)$ then the maximal spectral type of $V($ and $W)$ is singular.

Final step. We deduce from $(B 2)$ and $(B 3)$ that $\mathcal{C}_{i, j}^{\prime \prime}:=\mathcal{C}_{i, j} \otimes L^{2}(X, \mu)$ is a $U_{S \times T^{-}}$ cyclic subspace for each pair $i, j$. Furthermore, the unitary operators $U_{S \times T}\left\lceil\mathcal{C}_{i, j}^{\prime \prime}\right.$ and $U_{S \times T} \uparrow \mathcal{C}_{i^{\prime}, j^{\prime}}^{\prime \prime}$ are unitarily equivalent if $i=i^{\prime}$ or spectrally disjoint if $i \neq i^{\prime}$. Hence $\mathcal{M}(S \times T)=M^{\prime}$. Next, it follows from Claim B that $\mathcal{M}(R \times S \times T)=M^{\prime}$. Then Claim $\mathrm{C}$ yields that the maximal spectral type of $U_{S \times T}$ is singular. Therefore if $B$ is a (probability preserving) Bernoulli shift then

$$
\mathcal{M}(S \times T \times B)=\mathcal{M}(S \times T) \cup\{\infty\}=M .
$$


It follows from $(B 4)$ and [FW] that the transformation $S \times T \times B$ is ergodic. Since the measure on the space of this transformation is non-atomic, the transformation is conservative.

It remains to consider the case where $M=\{\infty\}$. However this case is well known. We recall that an ergodic conservative infinite measure preserving $K$-transformation has Lebesgue spectrum of infinite multiplicity $[\mathrm{Pa}]$. Any such a transformation is mixing $[\mathrm{KS}]$.

Our next purpose is to prove Theorem 0.4. For that we need an auxiliary lemma from $[\mathrm{Ag}]$ and $[\mathrm{Ry} 2]$.

Lemma 3.2. Let $V$ be unitary operator with a simple spectrum in Hilbert space $\mathcal{H}$. Assume that for each $n \in \mathbb{N}$, there are a sequence $k_{t}^{(n)} \rightarrow \infty$ and reals $\alpha_{n}, \beta_{n}>0$ such that $V_{t}^{k_{t}^{(n)}} \rightarrow \alpha_{n} I+\beta_{n} V^{*}$ weakly as $t \rightarrow \infty$, and $\alpha_{1}, \alpha_{2}, \ldots$ are pairwise different. Then $\exp (V)$ has a simple spectrum.

We note that $\exp (V)$ has a simple spectrum if and only if each symmetric power $V^{\odot n}$ has a simple spectrum and the convolution powers of a measure of maximal spectral types for $V$ are all mutually singular.

Proof of Theorem 0.4. We only sketch the idea of the proof. As in the proof of Theorem 0.1 we will use the forcing mixing technique. For that construct inductively a sequence of $\left(1-\alpha_{n}-\beta_{n}\right)$-partially high staircases $T_{n}$ such that the weak closure of the powers of $U_{T_{n}}$ contains $\alpha_{n} I+\beta_{n} U_{T_{n}}^{*}$ with $\alpha_{n}, \beta_{n} \rightarrow 0$ as $n \rightarrow \infty$. By Lemma 3.2, all the symmetric powers $U_{T_{n}}^{\odot k}$ have a simple spectrum, $k=1,2, \ldots$ Let $T_{n}$ be associated with a sequence $\left(C_{k}^{(n)}, F_{k-1}^{(n)}\right)_{k \geq 1}$. Then we construct a $(C, F)$ transformation $T$ associated to

$$
F_{0}^{(1)}, C_{1}^{(1)}, \ldots, F_{k_{1}}^{(1)}, C_{1}^{(2)}, F_{1}^{(2)} \ldots, F_{k_{2}}^{(2)}, \ldots,
$$

where the stopping times $k_{1}, k_{2}, \ldots$ are chosen in such a way to retain the simple spectrum of the symmetric powers "in the limit", i.e. for $T$. This is possible because the property to have a simple spectrum is approximable: if a unitary operator admits a sequence of cyclic spaces appoximating the entire space then the operator has a simple spectrum. We note that $T$ is an almost high staircase since $\alpha_{n}, \beta_{n} \rightarrow 0$. Hence it is conservative and ergodic. Moreover, we can satisfy the restricted growth condition. Hence $T$ is mixing by Theorem 0.2 .

\section{Applications to Poisson suspensions}

Let $(X, \mathfrak{B})$ be a standard Borel space and let $\mu$ be an infinite $\sigma$-finite non-atomic measure on $\mathfrak{B}$. Fix an increasing sequence of Borel subsets $X_{1} \subset X_{2} \subset \cdots$ with $\bigcup_{i>0} X_{i}=X$ and $\mu\left(X_{i}\right)<\infty$ for each $i$. A Borel subset is called bounded if it is contained in some $X_{i}$. Let $\tilde{X}_{i}$ denote the space of finite measures on $X_{i}$. For each bounded subset $A \subset X_{i}$, let $N_{A}$ stand for the map

$$
\widetilde{X}_{i} \ni \omega \mapsto \omega(A) \in \mathbb{R} .
$$

Denote by $\widetilde{\mathfrak{B}}_{i}$ the smallest $\sigma$-algebra on $\widetilde{X}_{i}$ in which all the maps $N_{A}, A \in \mathfrak{B} \cap X_{i}$, are measurable. It is well known that $\left(\widetilde{X}_{i}, \widetilde{\mathfrak{B}}_{i}\right)$ is a standard Borel space. Denote by $(\widetilde{X}, \widetilde{\mathfrak{B}})$ the projective limit of the sequence

$$
\left(\widetilde{X}_{1}, \widetilde{\mathfrak{B}}_{1}\right) \leftarrow\left(\widetilde{X}_{2}, \widetilde{\mathfrak{B}}_{2}\right) \leftarrow \cdots
$$


where the arrows denote the (Borel) natural restriction maps. Then $(\widetilde{X}, \widetilde{\mathfrak{B}})$ is a standard Borel space. To put it in other way, $\widetilde{X}$ is the space of measures on $X$ which are $\sigma$-finite along $\left(X_{i}\right)_{i>0}$. We define a probability measure $\widetilde{\mu}$ on $(\widetilde{X}, \widetilde{\mathfrak{B}})$ by the following two conditions:

(i) $\tilde{\mu} \circ N_{A}^{-1}=e^{-\mu(A)} \sum_{n=0}^{\infty} \frac{\mu(A)^{n}}{n !} \delta_{n}$ for each bounded subset $A$, where $\delta_{n}$ is the Dirac measure on $\mathbb{R}$ supported at $n$,

(ii) if $A$ and $B$ are disjoint bounded subsets of $X$ then the random variables $N_{A}$ and $N_{B}$ on $(\widetilde{X}, \widetilde{\mathfrak{B}}, \widetilde{\mu})$ are independent.

These conditions determine $\widetilde{\mu}$ in a unique way. Let $T$ be a Borel invertible transformation $T$ such that $T$ and $T^{-1}$ preserve the subclass of bounded subsets. If $T$ preserves $\mu$ then it induces a Borel isomorphism $\widetilde{T}$ of $\widetilde{X}$ by the formula $\widetilde{T} \widetilde{\omega}:=\widetilde{\omega} \circ T$. We recall that the dynamical system $(\widetilde{X}, \widetilde{\mathfrak{B}}, \widetilde{\mu}, \widetilde{T})$ is called the Poisson suspension of $(X, \mathfrak{B}, \mu, T)$ [CFS]. A probability preserving transformation is called a Poissonian automorphism if it is isomorphic to the Poissonian suspension of an infinite measure preserving transformation. If $T$ has no invariant subsets of positive and finite measure then $\widetilde{T}$ is weakly mixing.

We state some new spectral realization problems:

(P1) which spectral multiplicities ${ }^{1}$ are realizable on weakly mixing Poissonian automorphisms?

(P2) which spectral multiplicities are realizable on mixing Poissonian automorphisms?

The same questions can be also asked for the Gaussian automorphisms. Since each Poissonian automorphism is spectrally equivalent to some Gaussian one, any (partial) solution of (P1) or (P2) is also a solution in the class of Gaussian automorphisms. It is unclear whether the converse holds.

It is known that $\mathcal{M}(T)$ is either $\{1\}$ or infinite for each Gaussian (and therefore Poissonian) automorphism [CFS]. Recall that the first mixing Gaussian automorphism with a simple spectrum appeared in [New].

Since $U_{\widetilde{T}}=\exp \left(U_{T}\right)[\mathrm{Ne}]$, Theorem 0.4 implies the existence of a mixing Poissonian automorphism $\widetilde{T}$ with a simple spectrum (Corollary 0.5 ). It is worth to note that $\widetilde{T}$ possesses some other interesting properties: if an invertible transformation $S$ preserves $\widetilde{\mu}$ and commutes with $\widetilde{T}$ then $S=\widetilde{R}$ for a $\mu$-preserving transformation $R$ commuting with $T$ [Ro2]. Also, $\widetilde{T}$ does not split into a Cartesian product of two other transformations [Ro2]. We note that the existence of non-mixing Poissonian automorphisms with a simple spectrum was mentioned in [Ro2].

Example 4.2. We will show that for each $p>1$, the semigroup $\left\{p, p^{2}, p^{3}, \ldots\right\}$ is realizable as the set of spectral multiplicities for a mixing Poissonian automorphism. Indeed, let $I_{p}$ stand for the identity transformation on $\mathbb{Z} / p \mathbb{Z}$. Let $T$ be a mixing infinite measure preserving transformation $T$ such that $\exp (T)$ has a simple spectrum (see Theorem 0.4). The Cartesian product $T \times I_{p}$ is no longer ergodic. However it is mixing. Hence the Poisson suspension $\widetilde{T \times I_{p}}$ is also mixing. It follows from $[\mathrm{Ry} 3]$ that $\mathcal{M}\left(\widetilde{T \times I_{p}}\right)=\left\{p, p^{2}, p^{3}, \ldots\right\}$.

\footnotetext{
${ }^{1}$ In the finite measure preserving case we consider the spectral multiplicities of operators restricted to the orthocomplement to the constants.
} 
Infinite "non-semigroups" of positive integers also can appear as Poissonian multiplicities.

Example 4.3. Let $T$ be as in Example 4.2. Then the transformation $T \odot T$ is mixing and therefore its Poissonian suspension $\widetilde{T \odot T}$ is also mixing. Moreover,

$$
U_{\widetilde{T \odot T}}=\exp \left(U_{T \odot T}\right)=\exp \left(U_{T} \odot U_{T}\right)=\bigoplus_{n=0}^{\infty}\left(U_{T} \odot U_{T}\right)^{\odot n}
$$

Since $\mathcal{M}\left(\left(U_{T} \odot U_{T}\right)^{\odot n}\right)=\left\{(2 n) ! /\left(2^{n} n !\right)\right\}$ and the measure of spectral types of $\left(U_{T} \odot U_{T}\right)^{\odot n}$ are pairwise disjoint, we obtain that

$$
\mathcal{M}(\widetilde{T \odot T})=\left\{\frac{(2 n) !}{2^{n} n !} \mid n \in \mathbb{N}\right\}=\{1,3,3 \cdot 5,3 \cdot 5 \cdot 7, \ldots\}
$$

Instead of $\widetilde{T \odot T}$ one can also consider Poissonian suspensions of other natural factors $T^{\otimes k} / \Gamma$ of the product $T^{\otimes k}$, where $\Gamma$ is a subgroup of the full symmetric group $S_{k}$. We leave details to the reader.

\section{Concluding Remarks}

5.1. There exist other mixing infinite measure preserving constructions which can be used in this paper instead of high staircases:

(o) High stochastic constructions with vanishing deterministic part. No principal difficulties arise to adapt Ornstein's random spacer techniques from [Or] to the infinite setup.

(o) Pure staircases and almost pure staircases with rapidly growing sequence of cuts. It follows from some unpublished results of the second named author that the restricted growth condition can be waived from the statement of Theorem 0.2 provided that $r_{n} / h_{n} \rightarrow 0$ and $r_{n} \rightarrow \infty$. If, in addition, $\sum_{n=1}^{\infty} r_{n} / h_{n}=\infty$ then the corresponding $(C, F)$-transformations are infinite measure preserving. In this case we can restrict ourself to the pure (and almost pure) staircases.

However, not striving for the full generality, we choose the high staircases satisfying the restricted growth condition as the most effective and fast way leading to the proof of Theorems $0.1-0.4$.

5.2. We note that the stopping times appearing in the proofs of Theorems 0.1 , 0.3 and 0.4 when we force mixing or ergodicity are not defined effectively. The same takes place in the other applications of the method to force mixing in $[\mathrm{Ag}],[\mathrm{Ry} 2]$, [Da4]. We hope to improve the method in a future work by developing an explicit effective algorithm of selecting the stopping times.

5.3. This remark is for aesthetically minded readers who may do not like the appearance of almost high staircases in the proof of Theorem 0.1. They will be pleased to know that the almost high staircases there can be replaced completely with high staircases (for the expense of some complication of the proof, of course). We now briefly outline the proof of this claim. Let $S$ be as in the proof of Theorem 0.1. In particular, $S^{i_{m}} \rightarrow I$ as $m \rightarrow \infty$. We will construct high staircase 
transformations $T_{n}$ such that for for some $L_{n}$,

$$
\begin{gathered}
U_{T_{n}}^{H_{q, k}^{(n)}} \rightarrow \mathcal{P}_{q}\left(U_{T_{n}}\right):=\frac{1}{q+1}\left(I+U_{T_{n}}^{-1}+\cdots+U_{T_{n}}^{-q}\right) \quad \text { as } k \rightarrow \infty \text { and } \\
\left(H_{q, k}^{(n)}\right)_{k=1}^{\infty} \text { is a subsequence of }\left(i_{m}\right)_{m=1}^{\infty}
\end{gathered}
$$

for each $q \geq L_{n}$. To satisfy (5-1), every number $q \geq L_{n}$ must occur in the sequence $\left(r_{k}^{(n)}\right)_{k=1}^{\infty}$ infinitely many times. The property (5-2) is satisfied by choosing $\left(z_{k}^{(n)}\right)_{k>0}$ in a right way. Now let $v_{S}$ be a $U_{S}$-cyclic vector in a cyclic subspace $\mathcal{C} \subset L^{2}(Y, \nu)$. Let $v_{T_{n}}$ be a $U_{T_{n}}$-cyclic vector in $L^{2}\left(X_{n}, \mu_{n}\right)$. Denote by $\widetilde{\mathcal{C}}$ the $U_{S} \otimes U_{T_{n}}$-cyclic space generated by $v_{S} \otimes v_{T_{n}}$. It follows from (5-1) and (5-2) that

$$
v_{S} \otimes \mathcal{P}_{q}\left(U_{T_{n}}\right) v_{T_{n}} \in \widetilde{\mathcal{C}} \quad \text { for all } q \geq L_{n}
$$

Hence

$$
(q+1) v_{S} \otimes \mathcal{P}_{q}\left(U_{T_{n}}\right) v_{T_{n}}-q v_{S} \otimes \mathcal{P}_{q-1}\left(U_{T_{n}}\right) v_{T_{n}}=v_{S} \otimes U_{T_{n}}^{-q} v_{T_{n}} \in \widetilde{\mathcal{C}}
$$

for each $q>L_{n}$. In particular, given any $p \in \mathbb{Z}$, we have $v_{S} \otimes U_{T_{n}}^{p-i_{m}} v_{T_{n}} \in \widetilde{\mathcal{C}}$ for all sufficiently large $m$. Hence $U_{S}^{i_{m}} v_{S} \otimes U_{T_{n}}^{p} v_{T_{n}} \in \widetilde{\mathcal{C}}$. Passing to the (weak) limit as $m \rightarrow \infty$, we obtain that $v_{S} \otimes U_{T_{n}}^{p} v_{T_{n}} \in \widetilde{\mathcal{C}}$. This yields

$$
\widetilde{\mathcal{C}}=\mathcal{C} \otimes L^{2}\left(X_{n}, \mu_{n}\right)
$$

as desired. Now to force mixing we must retain (5-3) in the limit as $n \rightarrow \infty$ for a representative family of $U_{S}$-cyclic subspaces $\mathcal{C}$. The sought-for high staircase $T$ appears as a certain limit of the sequence $\left(T_{n}\right)_{n>0}$. At the same time we must have $L_{n} \rightarrow \infty$ in order to obtain $r_{n} \rightarrow \infty$ for $T$. The rest of the argument is as in the proof of Theorem 0.1 .

\section{REFERENCES}

[Aa] J. Aaronson, An introduction to infinite ergodic theory, Amer. Math. Soc., Providence, R. I., 1997.

[Ad] T. M. Adams, Smorodinsky's conjecture on rank one systems, Proc. Amer. Math. Soc. 126 (1998), 739-744.

[AFS] T. Adams, N. Friedman and C.E. Silva, Rank-one power weak mixing nonsingular transformations, Erg. Th. \& Dyn. Sys. 21 (2001), 1321-1332.

[Ag] O. N. Ageev, Mixing with staircase multiplicity function, Ergodic Theory Dynam. Systems 28 (2008), 1687-1700.

[CK] J. Choksi and S. Kakutani, Residuality of ergodic measure transformations and of ergodic transformations which preserve an infinite measure, Indiana Univ. Math. J. 28 (1979), 453-469.

[CFS] I. P. Cornfeld, V. S. Fomin and Ya. G. Sinai, Ergodic theory, Springer, New York, 1982.

[Da1] A. I. Danilenko, Funny rank one weak mixing for nonsingular Abelian actions, Isr. J. Math. 121 (2001), 29-54.

[Da2] - Infinite rank one actions and nonsingular Chacon transformations, Illinois J. Math. 48 (2004), 769-786.

[Da3] , $(C, F)$-actions in ergodic theory, Geometry and Dynamics of Groups and Spaces, Progr. Math., vol. 265, 2008, pp. 325-351.

[Da4] N New spectral multiplicities for mixing transformations, preprint, arXiv:0908.1640. 
[DaR] A. I. Danilenko and V. V. Ryzhikov, Spectral multiplicities for infinite measure preserving transformations, Funct. Anal. Appl. (to appear).

[DaS1] A. I. Danilenko and C. E. Silva, Multiple and polynomial recurrence for Abelian actions in infinite measure, J. London Math. Soc. 69 (2004), 183-200.

[DaS2] - Ergodic theory: non-singular transformations, Encyclopedia of complexity and systems science, Springer, 2009, pp. 3055-3083.

[dJ] A. del Junco, A simple map with no prime factors, Isr. J. Math. 104 (1998), 301-320.

[FW] H. Furstenberg and B. Weiss, The finite multipliers of infinite ergodic transformations, The structure of attractors in dynamical systems, Lecture Notes in Math 668, Springer, Berlin, 1978, pp. 127-132.

[KS] U. Krengel and L. Sucheston, On mixing in infinite measure spaces., Z. Wahrscheinlichkeitstheorie und Verw. Gebiete 13 (1969), 150-164.

[Na] M. G. Nadkarni, Spectral theory of dynamical systems, Birkhäuser Advanced Texts: Basler Lehrbücher, Birkhäuser Verlag, Basel, 1998.

$[\mathrm{Ne}] \quad Y u$. Neretin, Categories of symmetries and infinite dimensional groups, Oxford University press, 1986.

[New] D. Newton, On Gausssian processes with simple spectrum, Z. Wahrscheinlichkeitstheorie Werv. Gebiete 5 (1966), 207-209.

[Or] D. S. Ornstein, On the root problem in ergodic theory, in Proc. Sixth Berkley Symp. Math. Stat. Prob. (Univ. California, Berkeley, Calif., 1970/1971), Vol II: Probability Theory, pp. 347-356. Univ. of California Press Berkeley, Calif., 1972.

[Pa] W. Parry, Ergodic and spectral analysis of certain infinite measure preserving transformations., Proc. Amer. Math. Soc. 16 (1965), 960-966.

[Ro1] E. Roy, Ergodic properties of Poissonian ID-processes, Ann. Probab. 35 (2007), 551-576.

[Ro2] , Poisson suspensions and infinite ergodic theory, Erg. Th. \& Dyn. Sys. 29 (2009), 667-683.

[Ry1] V. V. Ryzhikov, Homogeneous spectrum, disjointness of convolutions, and mixing properties of dynamical systems, Selected Russian Mathematics 1 (1999), 13-24.

[Ry2] W Weak limits of powers, the simple spectrum of symmetric products and mixing constructions of rank 1, Sb. Math. 198 (2007), 733-754.

[Ry3] - Spectral multiplicities and asymptotic operator properties of actions with invariant measure, Sb. Math. 200 (2009).

Max Planck Institute for Mathematics, Vivatsgasse 7, D-53111 Bonn, Germany

Permanent address: Institute for Low Temperature Physics \& Engineering of National Academy of Sciences of Ukraine, 47 Lenin Ave., Kharkov, 61164, UKRAine E-mail address: alexandre.danilenko@gmail.com

Department of Mechanics and Mathematics, Lomonosov Moscow State University, GSP-1, Leninskie Gory, Moscow, 119991, Russian Federation

E-mail address: vryzh@mail.ru 